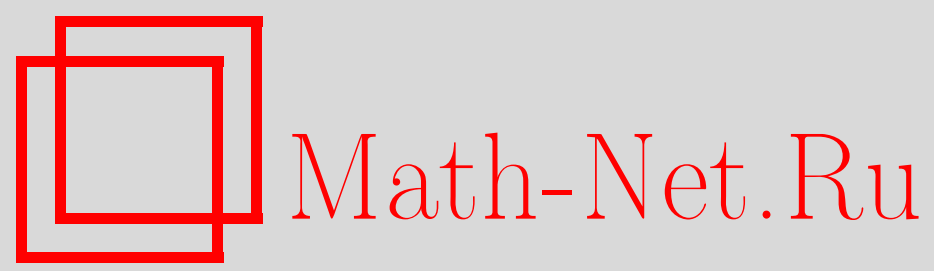

К. В. Сидоров, Н. Н. Филатова, П. Д. Шемаев, Н. И. Бодрина, Применение нечетких высказываний для интерпретации влияния эмоций на когнитивную активность человека, Нечеткие системы и мягкие вычисления, 2018, том 13, выпуск 2, 147-165

DOI: https://doi.org/10.26456/fssc47

Использование Общероссийского математического портала Math-Net.Ru подразумевает, что вы прочитали и согласны с пользовательским соглашением

http://www.mathnet.ru/rus/agreement

Параметры загрузки:

IP : 54.237 .59 .107

26 апреля 2023 г., 16:00:32 
УДК 004.853; 004.855.5

\section{ПРИМЕНЕНИЕ НЕЧЕТКИХ ВЫСКАЗЫВАНИЙ ДЛЯ ИНТЕРПРЕТАЦИИ ВЛИЯНИЯ ЭМОЦИЙ НА КОГНИТИВНУЮ АКТИВНОСТЬ ЧЕЛОВЕКА ${ }^{1}$}

Сидоров К.В., Филатова Н.Н., Шемаев П.Д., Бодрина Н.И. Тверской государственный технический университет, г. Тверь

Поступила в редакцию 06.11.2018, после переработки 20.12.2018.

В статье представлена модель когнитивной активности, позволяющая на основе нечетких оценок характеристик паттернов биомедицинских сигналов (электроэнцефалограмм и электромиограмм) отслеживать изменение когнитивной деятельности человека с учетом влияния эмоциональной стимуляции. Указанная задача решена путем формирования новой системы признаков, характеризующей спектры мощности биомедицинских сигналов. Созданный на основе нечетких множеств математический аппарат позволяет коррелированно интерпретировать когнитивную активность по паттернам электроэнцефалограмм и электромиограмм, а также формировать вербальные заключения о ее скорости и направлении развития.

Ключевые слова: нечеткое множество, когнитивная активность, эмоция, биотехническая система, биомедицинский сигнал, ЭМГ, ЭЭГ.

Нечеткие системы и мягкие вычисления. 2018. T. 13, № 2. С. 147-165. https://doi.org/10.26456/fssc47

\section{Введение}

В настоящее время мониторинг эмоционального состояния человека является актуальной задачей, связанной с прогнозированием его поведения и оценкой работоспособности [1-4]. Важным направлением исследований в этой области является определение степени влияния эмоций на когнитивную активность человека. В последние полтора-два десятилетия изучению вопроса взаимосвязи эмоционального состояния человека с эффективностью выполнения различных когнитивных задач уделяется большое внимание. Исследования можно условно разделить на две группы. В первом случае эмоциональный стимул и когнитивная задача объединены в один объект, т.е. сама задача имеет некий эмоциональный окрас. Чаще всего такие исследования связаны с изучением памяти $[5,6]$ и принятием решений [7-10]. Во вторую группу входят работы, где эмоциональный стимул предшествует этапу выполнения когнитивных задач, создавая, таким образом, эмоциональный настрой [11-14]. Довольно часто исследователи могут рассматривать данные процессы и по отдельности. В большинстве случаев это связано с высокой сложностью получения и интерпретации информации от различных

\footnotetext{
${ }^{1}$ Работа выполнена при финансовой поддержке РФФИ (проекты № 17-01-00742, № 18-3700225).
} 
физиологических подсистем организма человека, отражающих эмоциональное состояние и мыслительную деятельность. На настоящий момент времени для данной области существует потребность в расширении прикладного инструментария.

Для эмоциональной стимуляции человека могут быть использованы готовые базы видео стимулов (например, DEAP, HUMAINE, Film Stim) [2] или изображений (например, IAPS, GAPED) [12]. Для определения валентности и интенсивности эмоций обычно испытуемые делают самоотчет. Однако помимо субъективной оценки часто требуется иметь количественное представление степени эмоционального возбуждения и когнитивной загруженности мозга. Это особенно актуально, когда требуется непрерывный мониторинг состояния человека и прогнозирование его развития. Например, когда испытуемый длительное время фокусируется на выполнении однотипных задач, постепенно уставая и теряя концентрацию. Для этого можно использовать различные биомедицинские сигналы. Чаще всего для решения подобного рода задач используются биоэлектрические потенциалы головного мозга. Так, например, в работе [15] данная задача решается с применением методов связанных с событиями потенциалов (ССП). В качестве стимулов, вызывающих эмоциональную реакцию, авторы используют изображения. Отмечается, что наличие стимула оказывает влияние на мыслительную деятельность и это отражается на компонентах ССП.

В другой работе [16] для анализа влияния эмоциональной окрашенности изображений на результативность решения мнестических заданий используются спектры электроэнцефалограмм (ЭЭГ). Авторы отмечают, что валентность стимула может воздействовать на результативность выполнения поставленных задач, однако одного лишь ЭЭГ может быть недостаточно, поскольку четкого разделения сигналов, отражающих эмоциональное состояние и когнитивную деятельность, не установлено.

Ещё в одном исследовании [17] рассмотрен вопрос взаимодействия эмоциональных реакций и когнитивно-моторной подсистемы человека, отвечающей за скорость и точность выполнения определенных движений. Установлено, что эмоционально окрашенные изображения оказывают существенное влияние на скорость реакции. Но, помимо электроэнцефалографа, существует большое количество инструментов, которые могут быть использованы для анализа эмоциональной и мыслительной активности $[1,2,18,19]$. Среди них можно выделить такие биомедицинские сигналы как электромиограмма (ЭМГ), электрокардиогамма (ЭКГ), электроокулограмма (ЭОГ), магнитно-резонансная томограмма (MPT) и т.д. Цель наших исследований состоит в том, чтобы подобрать оптимальный набор средств для решения поставленных задач. Применяя несколько каналов регистрации, можно повысить точность работы создаваемых на их основе интерпретаторов. Нами проведены эксперименты с помощью биотехнической системы (БТС «EMG/EEG») [20-22], включающей методы электромиографии и электроэнцефалографии, которые позволяют получать объективную информацию о физиологическом и эмоциональном состояниях человека в виде коррелированных количественных данных, включающих показатели электрической активности мозга и регистрации мышечных потенциалов. Выдвинуты гипотезы о том, что ЭМГ-сигналы лучше передают эмоциональную реакцию испытуемого, поскольку для наблюдения могут использоваться независимые группы мышц, отдельно как для положительных, так и для отрицательных эмоций. ЭЭГ-сигналы используются для мониторинга когнитивной деятельности человека. 


\section{1. Методика постановки экспериментов}

Система «EMG/EEG» (Рис. 1) включает в себя два канала регистрации биомедицинских сигналов: ЭМГ и ЭЭГ. Система представляет собой аппаратнопрограммное средство, включающее электронейромиограф «Нейро-МВП-4» (ООО «Нейрософт», г. Иваново) и компьютерный электроэнцефалографанализатор ЭЭГА-21/26 «Энцефалан-131-03» (ООО НПКФ «Медиком МТД», г. Таганрог), подключенные к персональным компьютерам с соответствующим программным обеспечением.
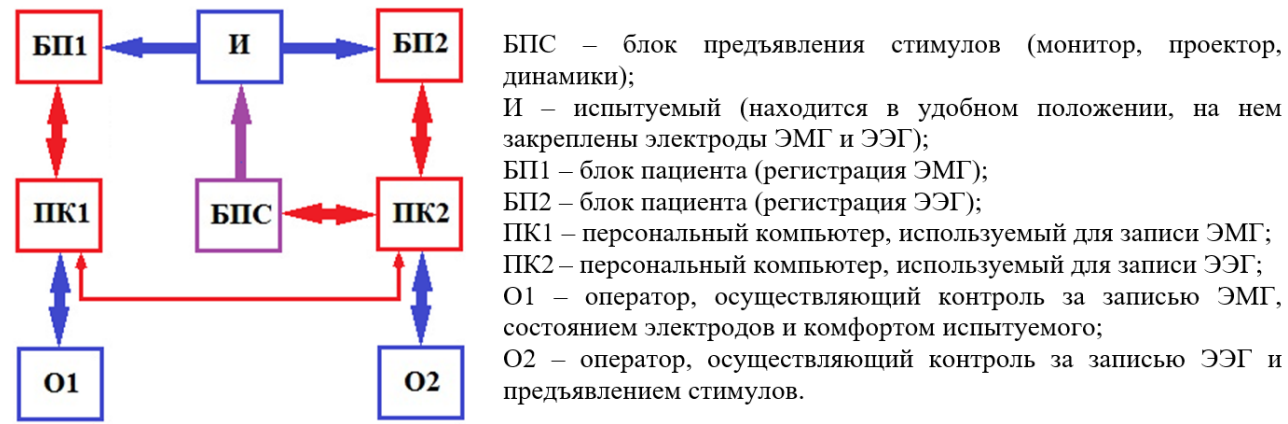

ПК1 - персональный компьютер, используемый для записи ЭМГ; ПК2 - персональный компьютер, используемый для записи ЭЭГ; O1 - оператор, осуществляющий контроль за записью ЭМГ, состоянием электродов и комфортом испытуемого;

О2 - оператор, осуществляющий контроль за записью ЭЭГ и предъявлением стимулов.

Pис. 1: Блок-схема биотехнической системы «EMG/EEG», предназначенной для мониторинга эмоций и когнитивной активности человека

При регистрации ЭЭГ показатели снимались по международной системе отведений «10-20» (Рис. 2, а) [23]. Регистрация производилась с применением комплекта ЭЭГ-электродов КЭ-ЭЭГ-10/20 «Энцефалан-КЭ» по 19 отведениям: О2A2, O1-A1, P4-A2, P3-A1, C4-A2, C3-A1, F4-A2, F3-A1, Fp2-A2, Fp1-A1, T6-A2, T5-A1, T4-A2, T3-A1, F8-A2, F7-A1, Pz-A1, Cz-A2, Fz-A1 (частота дискретизации 250 Гц).

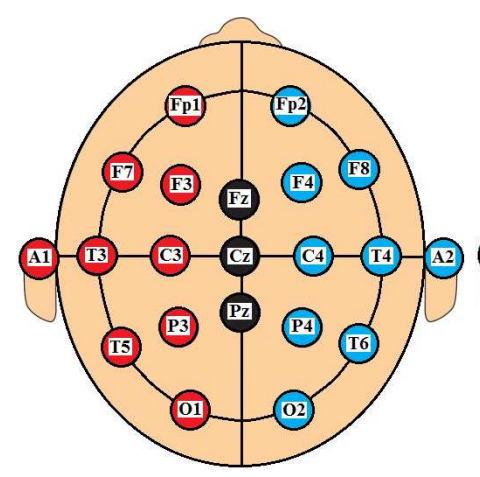

(a)



(б)

Рис. 2: Международная система отведений 10-20» для регистрации ЭЭГ

При регистрации ЭМГ-сигналов показатели снимались с помощью биполярных чашечковых $\mathrm{Ag} / \mathrm{AgCl}$ электродов диаметром 3 мм, закрепленных с левой стороны лица в области «corrugator supercilii» и «zygomaticus major» в соответствии с методикой «Fridlund and Сасіорро» (Рис. 3) [24]. Данная сторона, как 
утверждается в работе [25], сильнее передает изменения мимических мышц. Для усиления контакта кожа испытуемого была предварительно обезжирена абразивной пастой «Everi», а электроды заполнены клеящей пастой «Ten 20». Регистрация ЭМГ велась при частоте дискретизации 1000 Гц.

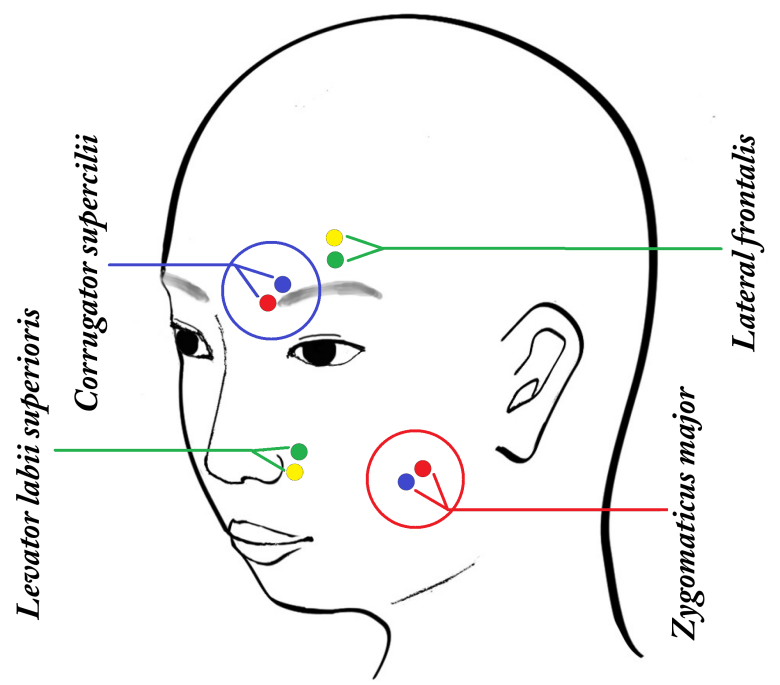

Puс. 3: Регистрация ЭМГ по методике «Fridlund and Cacioppo»

В качестве испытуемых выступили условно здоровые люди (студенты, аспиранты и сотрудники ТвГТУ). Всего в экспериментах участвовало 10 человек (5 женщин и 5 мужчин), возраст которых варьировался от 20 до 30 лет. Каждый испытуемый дал свое добровольное согласие на участие в проведении экспериментов, после того как был подробно проинструктирован по задачам и действиям, которые ему необходимо было выполнить. Эксперименты проводили в дневное время суток. Во время предъявления стимулов и при регистрации откликов (ЭЭГ и ЭМГ) испытуемые располагались в удобном кресле.

Для допуска к участию в экспериментах каждый испытуемый перед началом исследования проходил психологическое тестирование (методика САН [26]).

Методика проведения экспериментов представлена на Рис. 4. В качестве когнитивных задач испытуемым на экран монитора предъявлялись слайды с примерами умножения двухзначных чисел на однозначные (количеством по 10 штук в каждом слайде).

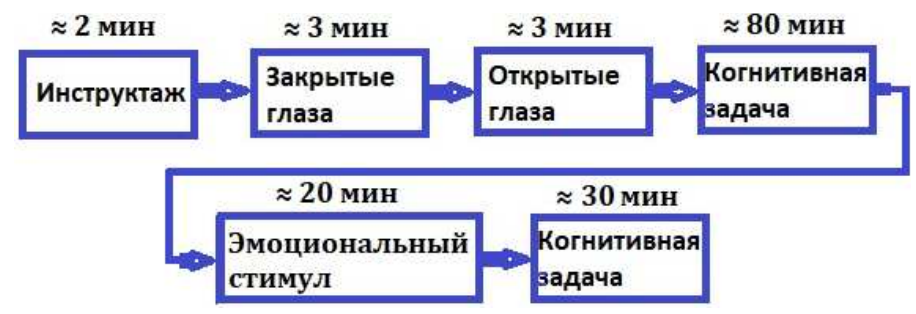

Рис. 4: Схема и время проведения экспериментов

Ориентировочно после 80 минут выполнения тестов запускалась эмоциональ- 
ная стимуляция в виде коротких позитивных видеоклипов (продолжительностью 20 минут), отобранных предварительно в соответствии с предпочтениями субъекта. Затем испытуемый переходил к завершающему этапу решения слайдов. Все ответы произносились вслух и записывались оператором. После эксперимента требовалось дать оценку эмоциональным стимулам по шкале: «Слабый», «Средний», «Сильный». Время проведения эксперимента выбрано таким образом, чтобы вызвать усталость у испытуемого и спровоцировать на ошибки.

С помощью предложенных БТС и методики проведен комплекс исследований, по результатам которых сформирована мультимодальная база данных (МБД), содержащая в себе безартефактные паттерны ЭМГ и ЭЭГ-сигналов. В рамках данной работы анализируются только те паттерны ЭЭГ и ЭМГ, которые соответствуют трем слайдам до стимуляции (проявление наибольшей усталости) и трем слайдам после эмоциональной стимуляции. Паттерны ЭЭГ и ЭМГ-сигналов отображают состояние испытуемого на одних и тех же интервалах времени.

\section{2. Модель описания ЭЭГ-сигналов}

Для паттернов ЭЭГ, продолжительностью по 4 секунды (1000 отсчетов), определялись спектральные признаки. В качестве разделяющих признаков применены отсчеты спектральной плотности мощности (СПМ), рассчитанные по методу Уэлча с использованием оконного быстрого преобразования Фурье (БПФ) [4]. Каждый объект описывается вектором вида $X=\left\{x_{1}, x_{2}, \ldots, x_{i}, \ldots, x_{r}\right\}$, где $x_{i}-$ ордината спектра мощности на частоте $f_{i}=\Delta f \bullet i ; x_{i}$ - значение $i$-го признака; $\Delta f$ - шаг по частоте; $\Delta f=f_{x} / F w ; f_{x}$ - частота дискретизации; $F w$ - ширина окна БПФ. Использовано окно преобразования Хемминга (ширина 256). Границы частотного диапазона составляют 0-125 Гц.

Описание для каждого объекта из МБД представляется в виде:

$$
X(s) \text { ЭЭГ }=<\left\{x_{1}, x_{2}, \ldots, x_{r}\right\}_{1},\left\{x_{1}, x_{2}, \ldots, x_{r}\right\}_{2}, \ldots,\left\{x_{1}, x_{2}, \ldots, x_{r}\right\}_{l}>\text {, }
$$

где $X(s)$ - векторы признаков СПМ; $s$ - номер объекта ЭЭГ; $s=1 \div 600 ; l-$ номер отведения ЭЭГ; $l=1 \div 19 ; r$ - номер признака СПМ; $r=1 \div 250$ (шаг расчета СПМ равен 0,5 Гц).

После определения вектора признаков (1) вычислялись абсолютные значения мощностей (AЗМ) - площадь под соответствующим участком СПМ по выбранным частотным диапазонам $\left(\mathrm{мkB}^{2} /\right.$ Гц $)[2]$.

Исследование ЭЭГ с помощью АЗМ продемонстрировало возможности локализации ритмов с наиболее информативными признаками. Наиболее информативными частотными диапазонами, иллюстрирующими варьирование спектра мощности при когнитивной деятельности, являются следующие ритмы: 1) дельта 1 - 0,5-2 Гц (Рис. 5, а); 2) дельта 2 - 2-4 Гц (Рис. 5, б).

Принято решение о целесообразности применения нового признака $\operatorname{Pr} i$ (Рис. 6), основанного на суммарной совокупности значений АЗМ (Рис. 2, б) по трем отведениям ЭЭГ:

$$
\begin{gathered}
\operatorname{Pr} 1=\sum(\mathrm{A} 3 \mathrm{M})_{F 3+F 4+F z}, \operatorname{Pr} 2=\sum(\mathrm{A} 3 \mathrm{M})_{C 3+C 4+C z} \\
\operatorname{Pr} 3=\sum(\mathrm{A} 3 \mathrm{M})_{P 3+P 4+P z} .
\end{gathered}
$$




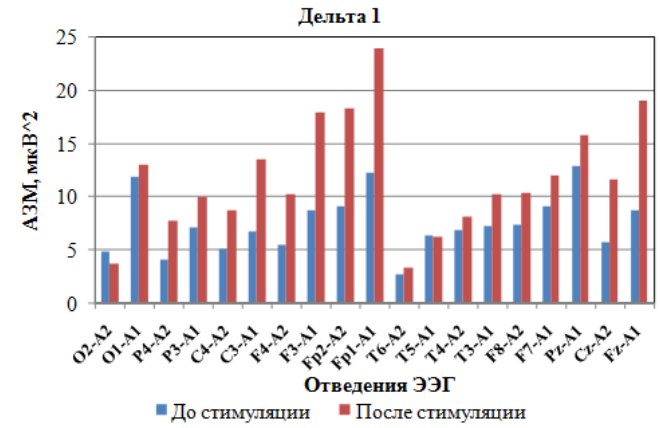

(a)



(б)

Рис. 5: Гистограмма разброса средних значений АЗМ в дельта-ритмах

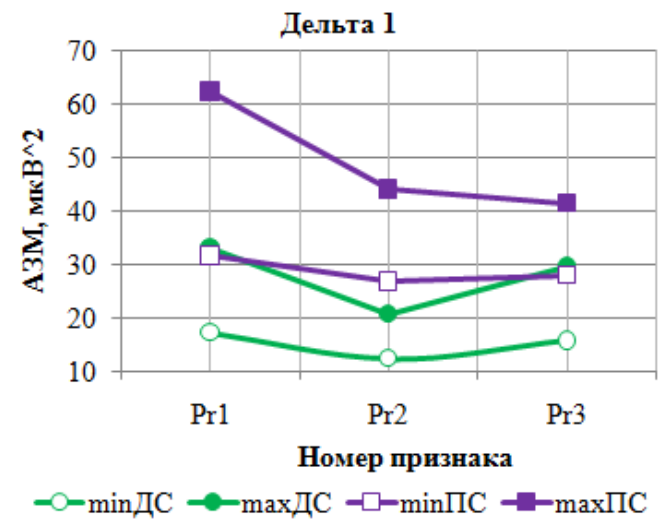

(a)

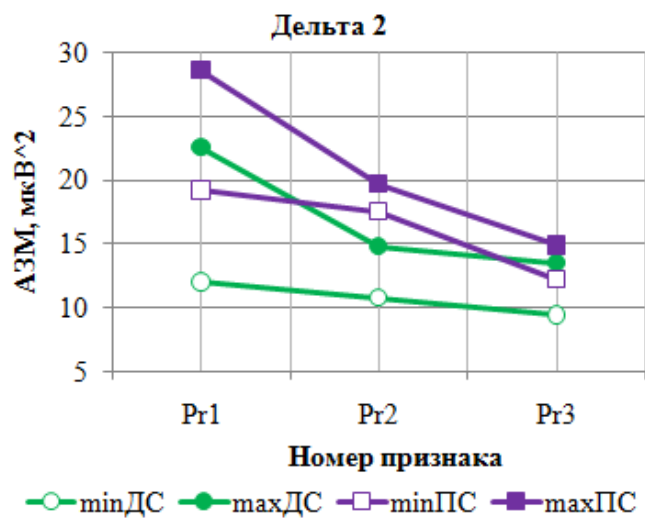

(б)

Рис. 6: Разброс средних значений АЗМ (ДС-до стимуляции, ПС-после стимуляции)

Новая система признаков (2) позволяет обеспечить хорошие уровни обобщения и уточнения описаний объектов ЭЭГ в приведенных состояниях (классах) когнитивной активности человека.

В результате анализа объектов из МБД, выявлена закономерность, заключающаяся в том, что после процедур эмоциональной стимуляции, у испытуемых наблюдается усиление значений признаков $\operatorname{Pr} i(2)$ в ритмах дельта 1 и дельта 2 (Рис. 7). Подобная ситуация характерна для всех испытуемых в диапазонах частот 0,5-2 Гц и 2-4 Гц.

На Рис. 8 продемонстрированы результаты изменения скорости (Рис. 8, а) и точности (Рис. 8, б) ответов испытуемых на предъявленные в слайдах когнитивные задачи. После эмоциональной стимуляции наблюдается тенденция уменьшения времени формулирования ответов, а также снижение количества ошибок, допущенных испытуемыми при выполнении когнитивных задач.

Таким образом, в качестве математической модели, описывающей паттерны ЭЭГ-сигналов, принято выражение $\mathrm{M}=<\operatorname{Pr} 1, \operatorname{Pr} 2, \operatorname{Pr} 3>$. 

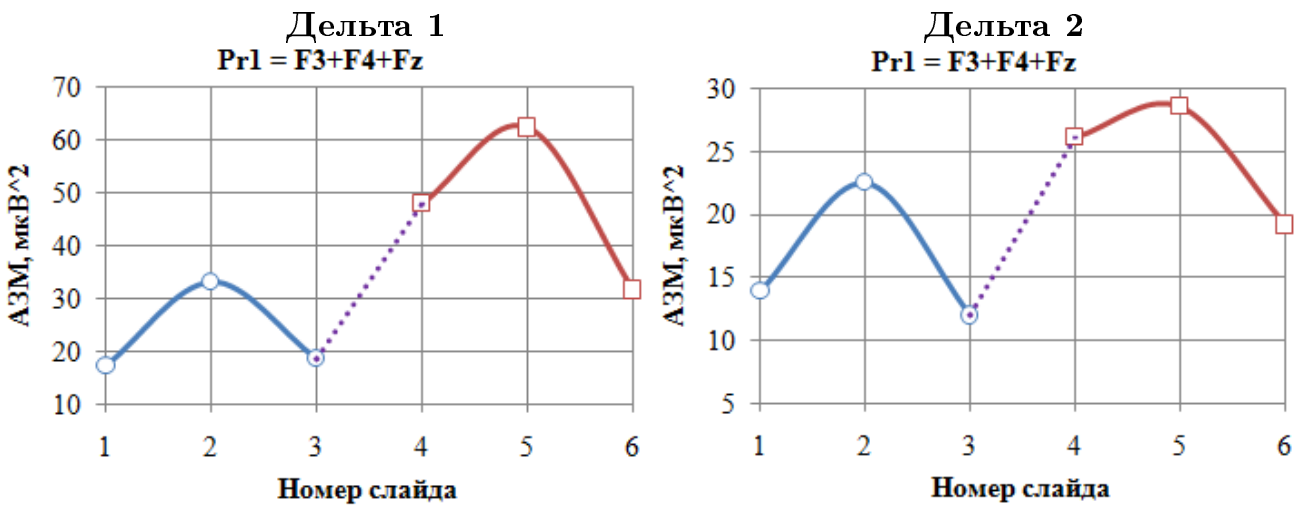

- -До стимуляции $\quad-\square-$ После стимуляции

- -До стимуляции $\quad-\square-$ После стимуляции

(a) информативный признак Pr1
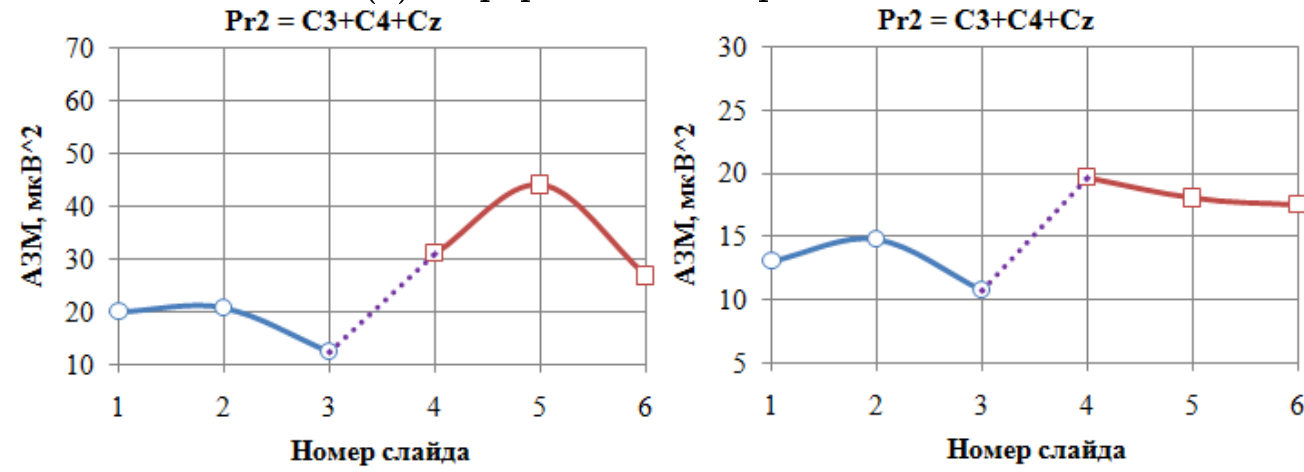

- -До стимуляции $\quad-\square-$ После стимуляции

- -До стимуляции $-\square-$ После стимуляции

(б) информативный признак Pr2
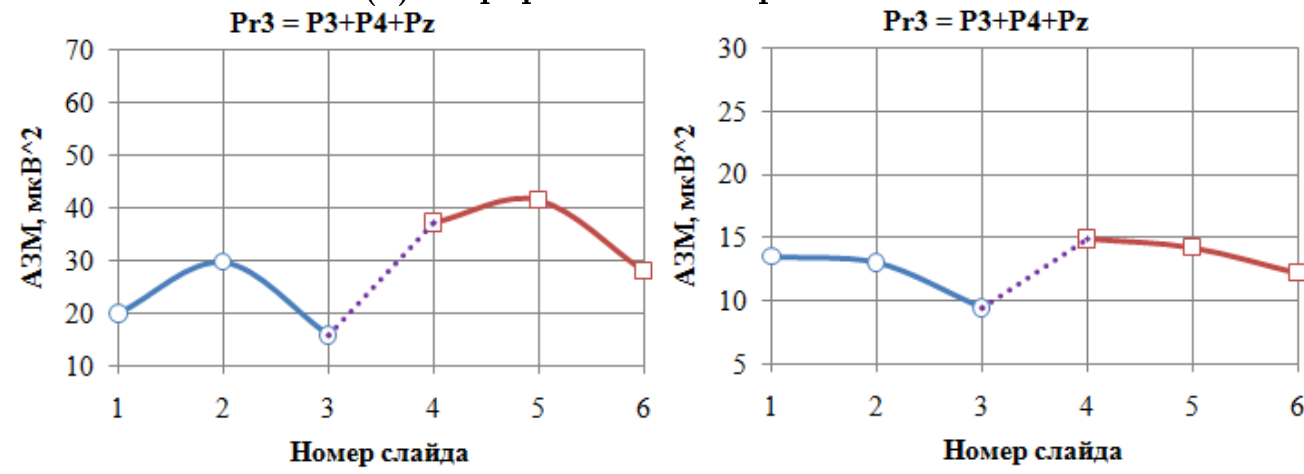

- -До стимуляции $-\square-$ После стимуляции

- -До стимуляции $\quad-\square-$ После стимуляции

(в) информативный признак Pr3

Pис. 7: Признаковое пространство $\operatorname{Pr}_{i}$ (слайды с когнитивными задачами до и после эмочиональной стимулячии)

\section{3. Модель описания ЭМГ-сигналов}

Полученные записи ЭМГ разбивались на фрагменты продолжительностью по 4 секунды. Для анализа использовались усредненные оценки амплитудного спек- 


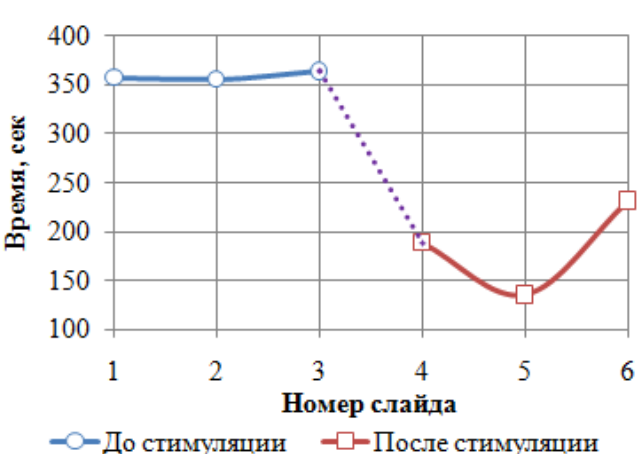

(a)

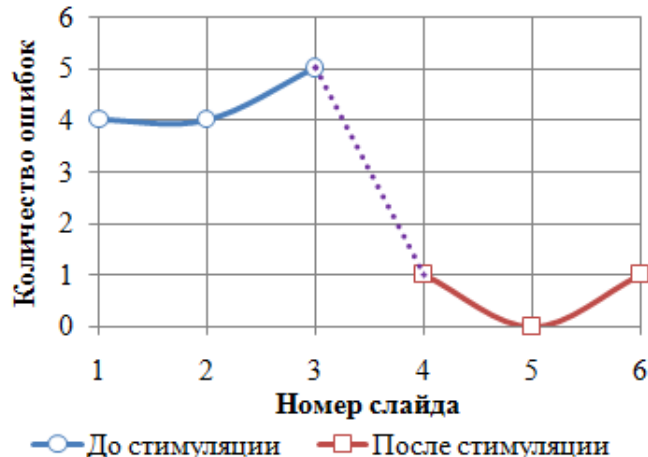

(б)

Рис. 8: Усредненные значения атрибутивных признаков (когнитивные задачи до и после эмоциональной стимуляции)

тра (fEMG, мкB) [25] по диапазону от 20 до 100 Гц. В данном случае нижняя граница обусловлена сильным влиянием мышечной активности (дыхание, моргание, движение глаз) на низких частотах. В то же время эксперименты с различными эмоциональными стимулами показали, что наибольшим изменениям подвержен участок спектра до 100 Гц.

На Рис. 9-10 представлены усредненные оценки признака fEMG и степень их разброса.

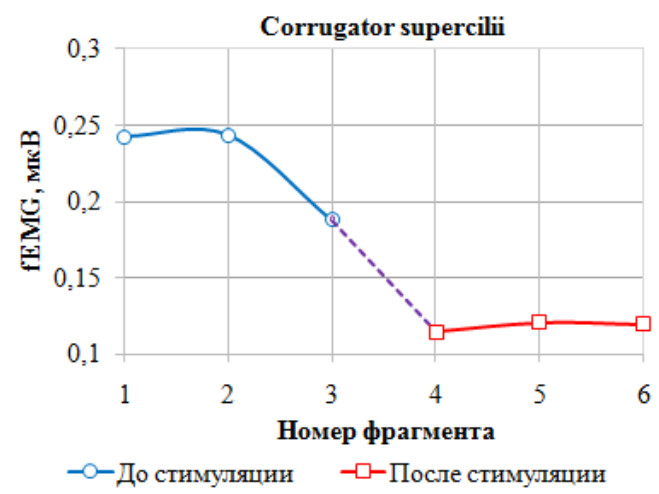

(a)

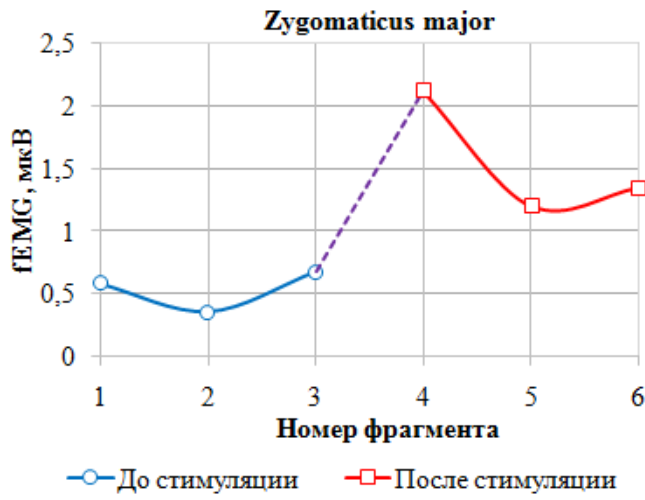

(б)

Рис. 9: Графики усредненных спектров ЭМГ-сигналов, зарегистрированных в точкаx: «corrugator supercilii» $u$ «zygomaticus major»

При выполнении когнитивных заданий наблюдается усиление спектральной активности сигнала, снимаемого от мышцы, сморщивающей бровь - «corrugator supercilii». После стимуляции данная характеристика снижается, т.е на определенный промежуток времени испытуемый расслабляется (Рис. 9, а). Параллельно, сигнал от мышцы, оттягивающей угол рта при улыбке - «zygomaticus major», усиливается после стимуляции, что может являться признаком изменения позитивного эмоционального состояния (Рис. 9, б).

Таким образом, в качестве модели, описывающей паттерны ЭМГ-сигналов, 


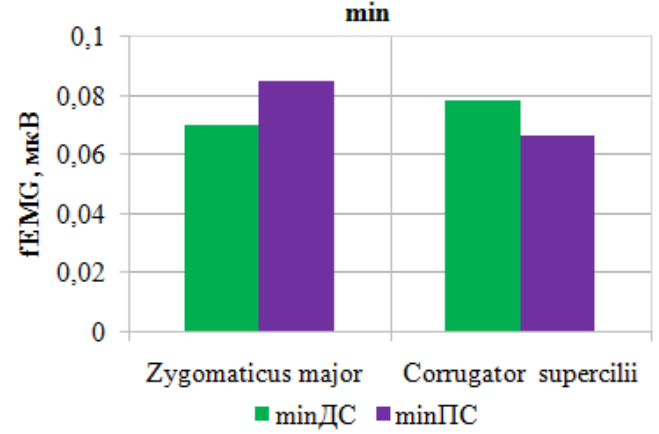

(a)

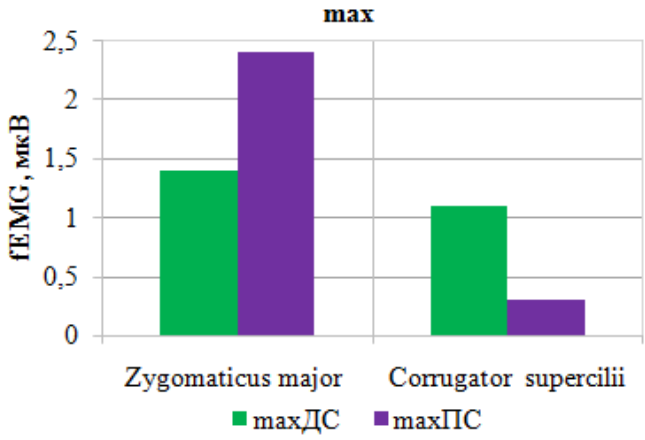

(б)

Рис. 10: Гистограммы разброса средних значений признака $f E M G$ (ДС - до стимуляции, ПС-после стимуляции)

принято выражение: $\mathrm{M}=<\mathrm{fEMG}_{C}, \mathrm{fEMG}_{M}>$, где $\mathrm{C}$ - канал «corrugator supercilii», Z - канал «zygomaticus major».

\section{4. Нечеткие оценки когнитивной активности человека по паттернам ЭЭГ-сигналов}

Модель когнитивной активности (MKA) на основе нечетких оценок характеристик паттернов ЭЭГ-сигналов позволяет отслеживать изменение когнитивной деятельности в течение времени. При ее создании мы исходили из двух требований: 1) модель должна быть ориентирована на интервальные оценки параметров ЭЭГ; 2) в состав модели должны быть включены только те компоненты, которые необходимы для вывода информации об активности человека в определенный момент времени $\left(t_{i}\right)$ и для прогнозирования ее изменения $\left(t_{i+d}\right)$.

Базовой основой алфавита для представления компонентов МКА являются суммарные признаки АЗМ по трем отведениям - Pr1, Pr2 и Pr3 (2). Учитывая существенную вариабельность оценок $\operatorname{Pr}_{i}$, в связи с индивидуальными различиями испытуемых, в MКА целесообразно использовать лингвистические переменные (ЛП) для описания основных характеристик когнитивной активности:

$\left[y_{1}:: \Pi_{1}\right]$ - лингвистическая переменная «сумма АЗМ Pr1», найденная по ритму дельта 1 ;

$\left[y_{2}, y_{3}:: Л_{2}, Л_{3}\right]$ - аналогичные лингвистические переменные «сумм АЗМ $\operatorname{Pr} 2$ и $\operatorname{Pr} 3 »$, рассчитанных для ритма дельта 1.

Для ритма дельта 2 ЛП применяются аналогично.

Исходя из вышеприведенного, формализованное представление МКА принимает следующий вид:

$$
\begin{gathered}
\mathrm{MKA}=\left\langle\left\{\operatorname{Pr} 1,\left\{\operatorname{TPr} 1_{-} j\right\}, \mu(\operatorname{TPr} 1) ; \operatorname{Pr} 2,\left\{\operatorname{TPr} 2_{-} j\right\}, \mu(\operatorname{TPr} 2) ;\right.\right. \\
\left.\left.\operatorname{Pr} 3,\left\{T \operatorname{Pr} 3 \_j\right\}, \mu(\operatorname{TPr} 3)\right\}\right\rangle,
\end{gathered}
$$

где $\left\{\mathrm{TPr} i \_j\right\}$ - терм-множество для оценки признака $\operatorname{Pr} i\left(\right.$ малый» $\operatorname{Pr} i \_1$, «средний» $\operatorname{Pr} i \_2$, «большой» $\left.\operatorname{Pr} i \_3\right) ; i, j=1 \div 3 ; \mu$ (TPr $\left.i\right)$ - функции принадлежности нечетких подмножеств универсального множества оценок $\operatorname{Pr} i=A \div B([10 ; 65]-$ для ритма дельта $1 ;[5 ; 35]$ - для ритма дельта 2). 
Переход от значений базовых переменных (Pri) к соответствующим значениям лингвистических переменных (ЛП) (ЛП $\left.{ }_{i}:\left\{\operatorname{Pr} i \_j\right\}, \mu(\mathrm{TPr} i)\right)$ осуществлен по специально построенным функциям принадлежностей $(\Phi П)$, отражающим мнения экспертов и результаты исследований (Рис. 11).

MKA (3) настраивается на образцы произвольной длительности с помощью ограничений на универсальное множество оценок признака $\operatorname{Pr} i=A \div B$, характеризующее суммарное значение АЗМ в ритмах дельта 1 и дельта 2.

Процедура фазификации признака Pri осуществляется с использованием треугольной конормы:

$$
\begin{gathered}
\max \left(\mu_{A}, \mu_{B}\right)=A \cup B \\
\max \left\{\mu_{{\text {PPr } i_{-1}}}(\text { Pri }), \mu_{\text {PPr }_{i_{-} 2}}(\text { Pri }), \mu_{T P r_{i_{-} 3}}(\text { Pri })\right\} \rightarrow \cup T P r i \_j, j=1 \div 3 .
\end{gathered}
$$

Составлены правила, которые позволяют определять принадлежность описания паттернов ЭЭГ-сигналов классам когнитивной активности человека: «низкая активность» $\left(L_{1}\right)$, «средняя активность» $\left(L_{2}\right)$ и «высокая активность» $\left(L_{3}\right)$ (Таблица 1).

Таблица 1: Фрагмент правил интерпретации когнитивной активности по

\begin{tabular}{|c|c|c|}
\hline $\mathrm{N}$ & $\begin{array}{l}\text { Нечеткие высказывания, определяющие } \\
\text { характерные признаки классов }\end{array}$ & $\begin{array}{l}\text { Класс } \\
\text { когнитивной активности }\end{array}$ \\
\hline 1 & $\pi \Pi_{1}=\operatorname{Pr} 1_{-} 1 \wedge \Omega \Pi_{2}=\operatorname{Pr} 2 \_1 \wedge \Omega \Pi_{3}=\operatorname{Pr} 3 \_1$ & $\begin{array}{l}L_{1}=D_{1}:: \ll \text { низкая актив- } \\
\text { ность» }\end{array}$ \\
\hline 2 & $\pi \Pi_{1}=\operatorname{Pr} 1 \_2 \wedge \Omega \Pi_{2}=\operatorname{Pr} 2 \_2 \wedge \pi \Pi_{3}=\operatorname{Pr} 3 \_2$ & $\begin{array}{l}L_{2}=D_{2}:: \ll \text { средняя актив- } \\
\text { ность» }\end{array}$ \\
\hline 3 & $\mathrm{I \Pi}_{1}=\operatorname{Pr} 1 \_3 \wedge Л \Pi_{2}=\operatorname{Pr} 2 \_3 \wedge Л \Pi_{3}=\operatorname{Pr} 3 \_3$ & $\begin{array}{l}L_{3}=D_{3}:: \ll \text { высокая актив- } \\
\text { ность» }\end{array}$ \\
\hline
\end{tabular}
ритмам дельта 1 и дельта 2

Для решения задачи распознавания классов когнитивной активности применялся алгоритм Mamdani $[27,28]$. Решением задачи распознавания класса когнитивной активности для выделенного ритма будет четкое высказывание (наименование класса $\left.L_{j}=D_{j}, \mu_{P R j}=\min \right)$, являющееся заключением в правиле с минимальным значением истинности всех его подусловий.

Окончательное решение задачи распознавания класса когнитивной активности формируется на основе результатов применения правил (табл. 1) для всех ритмов ЭЭГ-сигнала. Для этого набор правил дополнен импликациями следующего вида:

1. Если по всем трем ЛП определяется один класс, например $\left(L_{f}\right)$, то его наименование используется для определения итогового класса активности $\left(\mathrm{MKA}=L_{f}\right)$. Степень соответствия этого заключения принимается равной максимальной ФП нечетких множеств, построенных для трех ЛП.

2. Если по двум ЛП (ЛП, ЛП $\left.{ }_{j}\right)$ определяется один класс, например $\left(L_{g}\right)$, а по одной ЛП $\left(Л_{k}\right)$ распознается другой класс, например $\left(L_{v}\right)$, то для определения активности используется наименование класса $L_{g}\left(\mathrm{MKA}=L_{g}\right)$. Степень соответствия этого заключения принимается равной минимальной ФП нечетких множеств, соответствующих двум ЛП. 


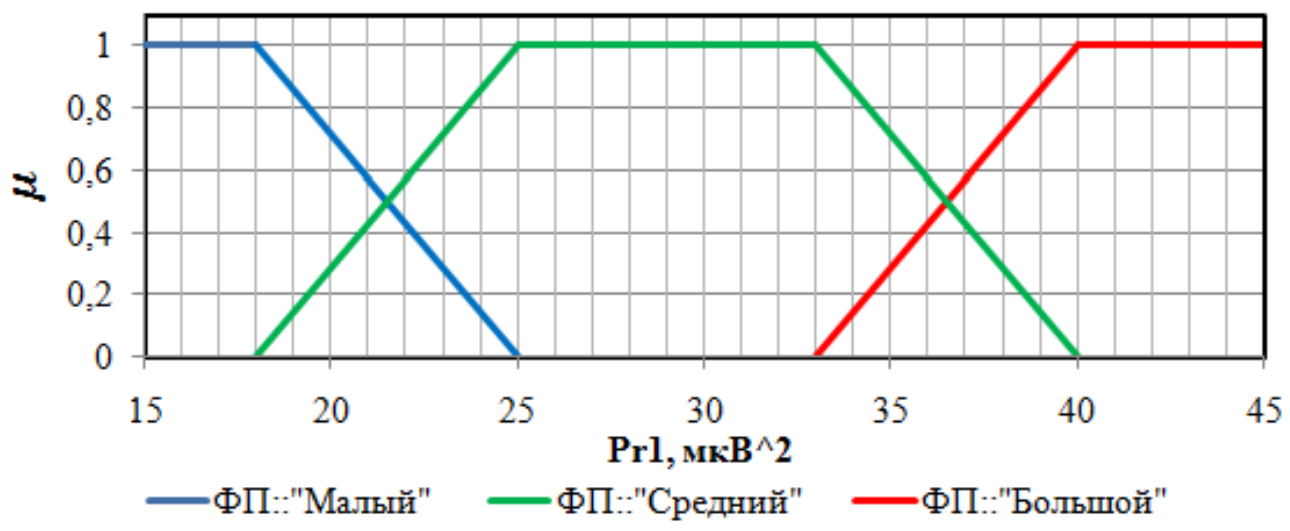

(a) базовая переменная $\operatorname{Pr} 1$



(б) базовая переменная $\operatorname{Pr} 2$

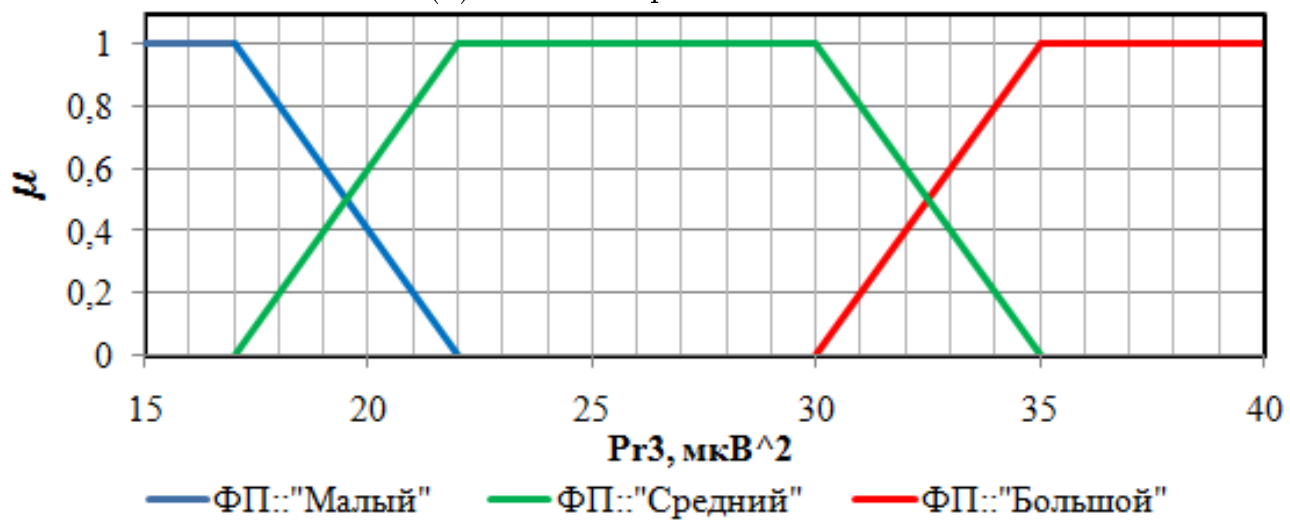

(в) базовая переменная $\operatorname{Pr} 3$

Puc. 11: ФП к термам $\{$ Pri $\}$ (ЛП $-Л \Pi_{3}$ для ритма дельта 1)

3. Если по всем трем ЛП распознаются разные классы, например $\left(L_{f}, L_{g}, L_{v}\right)$, то следует повторить процедуру интерпретации, используя другой паттерн ЭЭГ-сигнала.

MKA на основе нечетких оценок характеристик для паттернов ЭМГ-сигналов 
настраивается аналогичным образом, в качестве базовых шкал выступают значения признака fEMG по каналам «corrugator supercilii» и «zygomaticus major».

\section{Заключение}

Предложенные методика и биотехническая система «EEG/EMG» позволяют получать и анализировать объективную информацию о физиологическом и эмоциональном состояниях человека в процессе когнитивной активности, а также исследовать влияние эмоциональной стимуляции на процесс его мышления и принятия решений. Полученные результаты являются предварительными и будут уточняться в процессе постановки следующих новых экспериментов.

Созданный на основе нечетких множеств математический аппарат позволяет коррелированно интерпретировать когнитивную активность человека по паттернам ЭМГ и ЭЭГ-сигналов, а также формировать вербальные заключения о ее скорости и направлении развития.

Дальнейшее направление исследований видится в расширении модели когнитивной активности новыми свойствами (в частности, оценкой тренда развития когнитивной деятельности).

\section{Список литературы}

[1] Rangayyan R.M. Biomedical signal analysis. 2nd edition. New York: Wiley-IEEE Press, 2015. 720 p. https://doi.org/10.1002/9781119068129

[2] Koelstra S. Affective and implicit tagging using facial expressions and electroencephalography: PhD Thesis. London: Queen Mary University of London, 2012. 167 p.

[3] Рабинович М.И., Мюезинолу М.К. Нелинейная динамика мозга: эмоции и интеллектуальная деятельность // Успехи физических наук. 2010. Т. 180, № 4. C. 371-387. https://doi.org/10.3367/UFNe.0180.201004b.0371

[4] Филатова Н.Н., Сидоров К.В. Компьютерные модели эмоций: построение и методы исследования. Тверь: Тверской государственный технический университет, 2017. 200 с.

[5] Kensinger E.A., Corkin S. Memory enhancement for emotional words: are emotional words more vividly remembered than neutral words? // Memory and Cognition. 2003. Vol. 31, № 8. Pp. 1169-1180. http://doi.org/10.3758/BF03195800

[6] Ray R.D., Zald D.H. Anatomical insights into the interaction of emotion and cognition in the prefrontal cortex // Neuroscience and Biobehavioral Reviews. 2012. Vol. 36, № 1. Pp. 479-501. https://doi.org/10.1016/j.neubiorev.2011.08.005

[7] Morewedge C.K., Buechel E.C. Motivated underpinnings of the impact bias in affective forecasts // Emotion. 2013. Vol. 13, № 6. Pp. 1023-1029. https://doi.org/10.1037/a0033797

[8] Fortin P.E., Cooperstock J.R. Laughter and tickles: toward novel approaches for emotion and behavior elicitation // IEEE Transactions on Affective Computing. 2017. Vol. 8, № 4. Pp. 508-521. https://doi.org/10.1109/TAFFC.2017.2757491 
[9] Lokannavar S., Lahane P., Gangurde A., Chidre P. Emotion recognition using EEG signals // International Journal of Advanced Research in Computer and Communication Engineering. 2015. Vol. 4, № 5. Pp. 54-56.

[10] Филатова Н.Н., Сидоров К.В., Терехин С.А. Программный комплекс для интерпретации невербальной информации путем анализа образцов речи или электроэнцефалограммы // Программные продукты и системы. 2015. Т. 3. C. $22-27$.

[11] Basso M.R., Schefft B.K., Ris M.D., Dember W.N. Mood and global-local visual processing // Journal of the International Neuropsychological Society. 1996. Vol. 2, № 3. Pp. 249-255. https://doi.org/10.1017/S1355617700001193

[12] Liu Y., Sourina O., Nguyen M.K. Real-Time EEG-based emotion recognition and its applications // Transactions on Computational Science XII. Eds. by M.L. Gavrilova, C.J.K. Tan, Sourin A., O. Sourina. Series: Lecture Notes in Computer Science. Vol. 6670. Berlin, Heidelberg: Springer. https://doi.org/10.1007/978-3642-22336-5_13

[13] Lan Z., Sourina O., Wang L., Liu Y. Real-time EEG-based emotion monitoring using stable features // The Visual Computer. 2016. Vol. 32, № 3. Pp. 347-358. https://doi.org/10.1007/s00371-015-1183-y

[14] Filatova N.N., Sidorov K.V., Shemaev P.D., Iliasov L.V. Monitoring attractor characteristics as a method of objective estimation of testee's emotional state // Journal of Engineering and Applied Sciences. 2017. Vol. 12, № 11. Pp. 9164-9175.

[15] Крутенкова Е.П., Есипенко Е.А., Рязанова М.К., Ходанович М.Ю. Влияние эмоциональных изображений на решение когнитивных задач // Вестник Томского государственного университета. Биология. 2013. № 1 (21). С. 129145 .

[16] Grissmann S., Faller J., Scharinger C., Spuler M, Gerjets P. Electroencephalography based analysis of working memory load and affective valence in an n-back task with emotional stimuli // Frontiers in Human Neuroscience. 2017. Vol. 11. ID 616. https://doi.org/10.3389/fnhum.2017.00616

[17] Lu Y., Jaquess K.J., Hatfield B.D., Zhou C., Li H. Valence and arousal of emotional stimuli impact cognitive-motor performance in an oddball task // Biological Psychology. 2017. Vol. 125. Pp. 105-114. https://doi.org/10.1016/j.biopsycho.2017.02.010

[18] Филатова Н.Н., Сидоров К.В. Интерпретация характеристик эмоций с помощью анализа аттракторов, реконструированных по ЭЭГ-сигналам // Нечеткие системы и мягкие вычисления. 2016. Т. 11, № 1. С. 57-76.

[19] Filatova N.N., Sidorov K.V., Shemaev P.D., Rebrun I.A. Emotion and Cognitive Activity Monitoring System // Proceedings of the 3rd RussianPacific Conference on Computer Technology and Applications, RPC 2018 (Russia, Vladivostok, August 18-25, 2018). IEEE, 2018. Pp. 166-169. https://doi.org/10.1109/RPC.2018.8482220 
[20] Filatova N.N., Sidorov K.V., Iliasov L.V. Automated system for analyzing and interpreting nonverbal information // International Journal of Applied Engineering Research. 2015. Vol. 10, № 24. Pp. 45741-45749.

[21] Filatova N.N., Sidorov K.V., Terekhin S.A., Vinogradov G.P. The system for the study of the dynamics of human emotional response using fuzzy trends // Proceedings of the First International Scientific Conference "Intelligent Information Technologies for Industry" (IITI'16). Eds. by A. Abraham, S. Kovalev, V. Tarassov, V. Snasel. Series: Advances in Intelligent Systems and Computing. Vol. 451. Cham: Springer, 2016. Pp. 175-184. https://doi.org/10.1007/978-3-319-33816-3_18

[22] Filatova N.N., Bodrina N.I., Sidorov K.V., Shemaev P.D. Organization of information support for a bioengineering system of emotional response research // Proceedings of the XX International Conference on Data Analytics and Management in Data Intensive Domains, DAMDID/RCDL 2018 (Russia, Moscow, October 9-12, 2018). Pp. 90-97.

[23] Jasper H.H. The ten-twenty electrode system of the international federation // Electroencephalography and Clinical Neurophysiology. 1958. Vol. 10. Pp. 371375 .

[24] Fridlund A.J., Cacioppo J.T. Guidelines for human electromyographic research // Psychophysiology. 1986. Vol. 23, № $5 . \quad$ Pp. 567-589. https://doi.org/10.1111/j.1469-8986.1986.tb00676.x

[25] Dimberg U., Petterson M. Facial reactions to happy and angry facial expressions: evidence for right hemisphere dominance // Psychophysiology. 2000. Vol. 37, № 5. Pp. 693-696. https://doi.org/10.1111/1469-8986.3750693

[26] Барканова О.В. Методики диагностики эмоциональной сферы. Красноярск: Литера-принт, 2009. 237 с.

[27] Mamdani E.H., Assilian S. An experiment in linguistic synthesis with a fuzzy logic controller // International Journal of Man-Machine Studies. 1975. Vol. 7, № 1. Pp. 1-13. https://doi.org/10.1016/S0020-7373(75)80002-2

[28] Mamdani E.H. Application of fuzzy logic to approximate reasoning using linguistic synthesis // IEEE Transactions on Computers. 1977. Vol. C-26, № 12. Pp. 1182-1191.

\section{Образец цитирования}

Сидоров К.В., Филатова Н.Н., Шемаев П.Д., Бодрина Н.И. Применение нечетких высказываний для интерпретации влияния эмоций на когнитивную активность человека // Нечеткие системы и мягкие вычисления. 2018. Т. 13, № 2. C. 147-165. https://doi.org/10.26456/fssc47 


\section{Сведения об авторах}

\section{1. Сидоров Константин Владимирович}

доцент кафедры Автоматизации технологических процессов Тверского государственного технического университета.

Россия, 170026, г. Тверь, набережная Афанасия Никитина, д. 22, ТвГТУ. E-mail: bmisidorov@mail.ru

\section{2. Филатова Наталья Николаевна}

профессор кафедры Автоматизации технологических процессов Тверского государственного технического университета.

Россия, 170026, г. Тверь, набережная Афанасия Никитина, д. 22, ТвГТУ. E-mail: nfilatova99@mail.ru

\section{3. Шемаев Павел Дмитриевич}

ассистент кафедры Автоматизации технологических процессов Тверского государственного технического университета.

Россия, 170026, г. Тверь, набережная Афанасия Никитина, д. 22, ТвГТУ. E-mail:ryuk-apple@yandex.ru

\section{4. Бодрина Наталья Ивановна}

доцент кафедры Автоматизации технологических процессов Тверского государственного технического университета.

Россия, 170026, г. Тверь, набережная Афанасия Никитина, д. 22, ТвГТУ. E-mail:vavilovani@mail.ru 


\title{
APPLICATION OF FUZZY STATEMENTS FOR INTERPRETATION OF THE EMOTIONAL INFLUENCE ON HUMAN COGNITIVE ACTIVITY
}

\author{
Sidorov Konstantin Vladimirovich \\ Associate Professor at Technological Processes Automation department, \\ Tver State Technical University \\ Russia, 170026, Tver, 22 Afanasy Nikitin Emb., TSTU. \\ E-mail: bmisidorov@mail.ru \\ Filatova Natalia Nikolaevna \\ Professor at Technological Processes Automation department, \\ Tver State Technical University \\ Russia, 170026, Tver, 22 Afanasy Nikitin Emb., TSTU. \\ E-mail: nfilatova99@mail.ru \\ Shemaev Pavel Dmitrievich \\ Assistant at Technological Processes Automation department, \\ Tver State Technical University \\ Russia, 170026, Tver, 22 Afanasy Nikitin Emb., TSTU. \\ E-mail: ryuk-apple@yandex.ru \\ Bodrina Natalia Ivanovna \\ Associate Professor at Technological Processes Automation department, \\ Tver State Technical University \\ Russia, 170026, Tver, 22 Afanasy Nikitin Emb., TSTU. \\ E-mail:vavilovani@mail.ru
}

Received 06.11.2018, revised 20.12.2018.

The article provides the model of cognitive activity, allowing to monitor changes in human cognitive performance considering influence of emotional stimulation, basing on fuzzy assessments of characteristics of patterns of biomedical signals (electroencephalography, electromyography). We formed a new system of attributes characterizing power spectrum of biomedical signals. This instrument based on fuzzy sets allows to correlatively interpret cognitive activity by electroencephalography and electromyography patterns and also create verbal conclusion about its speed and progress direction.

Keywords: fuzzy set, cognitive activity, emotion, bioengineering system, biomedical signal, EMG, EEG.

\section{Citation}

Sidorov K.V., Filatova N.N., Shemaev P.D., Bodrina N.I., "Application of fuzzy statements for interpretation of the emotional influence on human cognitive activity", Nechetkie Sistemy i Myagkie Vychisleniya [Fuzzy Systems and Soft Computing], 13:2 (2018), 147-165 (in Russian). https://doi.org/10.26456/fssc47 


\section{References}

[1] Rangayyan R.M., Biomedical signal analysis, 2nd edition, Wiley-IEEE Press, New York, 2015, 720 pp., https://doi.org/10.1002/9781119068129.

[2] Koelstra S., Affective and implicit tagging using facial expressions and electroencephalography, PhD Thesis, Queen Mary University of London, London, 2012, 167 pp.

[3] Rabinovich M.I., Muezzinoglu M.K., "Nonlinear dynamics of the brain: emotion and cognition", Physics-Uspekhi, 53:4 (2010), 357-372, https://doi.org/10.3367/UFNe.0180.201004b.0371.

[4] Filatova N.N., Sidorov K.V., Kompyuternye modeli emotsij: postroenie $i$ metody issledovaniya [Computer models of emotions: the construction and methods of research], Tver State Technical University, Tver, 2017 (in Russian), 200 pp.

[5] Kensinger E.A., Corkin S., "Memory enhancement for emotional words: are emotional words more vividly remembered than neutral words?", Memory and Cognition, 31:8 (2003), 1169-1180, http://doi.org/10.3758/BF03195800.

[6] Ray R.D., Zald D.H., "Anatomical insights into the interaction of emotion and cognition in the prefrontal cortex", Neuroscience and Biobehavioral Reviews, 36:1 (2012), 479-501, https://doi.org/10.1016/j.neubiorev.2011.08.005.

[7] Morewedge C.K., Buechel E.C., "Motivated underpinnings of the impact bias in affective forecasts", Emotion, 13:6 (2013), 1023-1029, https://doi.org/10.1037/a0033797.

[8] Fortin P.E., Cooperstock J.R., "Laughter and tickles: toward novel approaches for emotion and behavior elicitation", IEEE Transactions on Affective Computing, 8:4 (2017), 508-521, https://doi.org/10.1109/TAFFC.2017.2757491.

[9] Lokannavar S., Lahane P., Gangurde A., Chidre P., "Emotion recognition using EEG signals", International Journal of Advanced Research in Computer and Communication Engineering, 4:5 (2015), 54-56.

[10] Filatova N.N., Sidorov K.V., Terekhin S.A., "Software package for interpretation of nonverbal information by analyzing speech patterns or electroencephalogram", Programmnye Produkty $i$ Sistemy [Software and Systems], 3 (2015), 22-27 (in Russian).

[11] Basso M.R., Schefft B.K., Ris M.D., Dember W.N., "Mood and global-local visual processing", Journal of the International Neuropsychological Society, 2:3 (1996), 249-255, https://doi.org/10.1017/S1355617700001193.

[12] Liu Y., Sourina O., Nguyen M.K., "Real-Time EEG-based emotion recognition and its applications", Transactions on Computational Science XII. V.6670, Lecture Notes in Computer Science, eds. M.L. Gavrilova, C.J.K. Tan, Sourin A., O. Sourina, Springer, Berlin, Heidelberg, https://doi.org/10.1007/978-3-642-223365_13.

[13] Lan Z., Sourina O., Wang L., Liu Y., "Real-time EEG-based emotion monitoring using stable features", The Visual Computer, 32:3 (2016), 347-358, https://doi.org/10.1007/s00371-015-1183-y. 
[14] Filatova N.N., Sidorov K.V., Shemaev P.D., Iliasov L.V., "Monitoring attractor characteristics as a method of objective estimation of testee's emotional state", Journal of Engineering and Applied Sciences, 12:11 (2017), 9164-9175.

[15] Krutenkova E.P., Esipenko E.A., Ryazanova M.K., Khodanovich M.Yu., "Emotional pictures impact on cognitive tasks solving", Vestnik Tomskogo Gosudarstvennogo Universiteta. Biologiya [Tomsk State University Journal of Biology], 2013, № 1 (21), 129-145 (in Russian).

[16] Grissmann S., Faller J., Scharinger C., Spuler M, Gerjets P., "Electroencephalography based analysis of working memory load and affective valence in an n-back task with emotional stimuli", Frontiers in Human Neuroscience, 11 (2017), 616, https://doi.org/10.3389/fnhum.2017.00616.

[17] Lu Y., Jaquess K.J., Hatfield B.D., Zhou C., Li H., "Valence and arousal of emotional stimuli impact cognitive-motor performance in an oddball task", Biological Psychology, 125 (2017), 105-114, https://doi.org/10.1016/j.biopsycho.2017.02.010.

[18] Filatova N.N., Sidorov K.V., "Interpretation of the emotion characteristics through the analysis of attractors reconstructed on EEG signals", Nechetkie Sistemy i Myagkie Vychisleniya [Fuzzy Systems and Soft Computing], 11:1 (2016), 57-76 (in Russian).

[19] Filatova N.N., Sidorov K.V., Shemaev P.D., Rebrun I.A., "Emotion and Cognitive Activity Monitoring System", Proceedings of the 3rd Russian-Pacific Conference on Computer Technology and Applications, RPC 2018 (Russia, Vladivostok, August 18-25, 2018), IEEE, 2018, 166-169, https://doi.org/10.1109/RPC.2018.8482220.

[20] Filatova N.N., Sidorov K.V., Iliasov L.V., "Automated system for analyzing and interpreting nonverbal information", International Journal of Applied Engineering Research, 10:24 (2015), 45741-45749.

[21] Filatova N.N., Sidorov K.V., Terekhin S.A., Vinogradov G.P., "The system for the study of the dynamics of human emotional response using fuzzy trends", Proceedings of the First International Scientific Conference "Intelligent Information Technologies for Industry" (IITI'16). V.451, Advances in Intelligent Systems and Computing, eds. A. Abraham, S. Kovalev, V. Tarassov, V. Snasel, Springer, Cham, 2016, 175-184, https://doi.org/10.1007/978-3-319-33816-3_18.

[22] Filatova N.N., Bodrina N.I., Sidorov K.V., Shemaev P.D., "Organization of information support for a bioengineering system of emotional response research", Proceedings of the XX International Conference on Data Analytics and Management in Data Intensive Domains, DAMDID/RCDL 2018 (Russia, Moscow, October 9-12, 2018), 90-97.

[23] Jasper H.H., "The ten-twenty electrode system of the international federation", Electroencephalography and Clinical Neurophysiology, 10 (1958), 371-375.

[24] Fridlund A.J., Cacioppo J.T., "Guidelines for human electromyographic research", Psychophysiology, 23:5 (1986), 567-589, https://doi.org/10.1111/j.14698986.1986.tb00676.x. 
[25] Dimberg U., Petterson M., "Facial reactions to happy and angry facial expressions: evidence for right hemisphere dominance", Psychophysiology, 37:5 (2000), 693-696, https://doi.org/10.1111/1469-8986.3750693.

[26] Barkanova O.V., Metodiki diagnostiki emotsionalnoj sfery [Methods of diagnosis of the emotional sphere], Litera-print, Krasnoyarsk, 2009 (in Russian), 237 pp.

[27] Mamdani E.H., Assilian S., "An experiment in linguistic synthesis with a fuzzy logic controller", International Journal of Man-Machine Studies, 7:1 (1975), 113, https://doi.org/10.1016/S0020-7373(75)80002-2.

[28] Mamdani E.H., "Application of fuzzy logic to approximate reasoning using linguistic synthesis", IEEE Transactions on Computers, C-26:12 (1977), 11821191. 